\title{
ROLE OF THE SURFACE ENERGY FOR THE FORMATION OF THE DOMAIN STRUCTURE AT MAGNETOELASTIC PHASE TRANSITION
}

\author{
H.V. GomonaJ* and V.M. LoKteV \\ National Technical University of Ukraine "KPI" \\ 37, Ave. Peremogy, 252056, Kyiv, Ukraine
}

The formation of the domain structure during magnetic ordering in the pure antiferromagnet with the rotational (non- $180^{\circ}$ ) domains can be treated as thermodynamically preferable if the free energy of the sample is minimized with the account of surface energy. The analogy between the role of magnetostatic energy in ferromagnets and surface energy of antiferromagnet is traced. The behavior of the domain structure and macroscopic strain tensor in the external magnetic field is investigated.

PACS numbers: $75.50 . \mathrm{Ee}, 75.60 . \mathrm{Ch}$

\section{Introduction}

In contrast to ferromagnets, the domain structure (DS) of pure antiferromagnets (AFM) that arises during magnetic ordering, is commonly considered as non-equilibrium, because of inevitable growth of free energy due to the energy of the domain walls. On the other hand, the experiments [1] unambiguously point to the reversible behavior of the domain structure of easy-plane AFM of $\mathrm{CoCl}_{2}$-type in the external magnetic field. In the present paper we propose the model that describes thermodynamically equilibrium DS of pure AFM with the rotational (non- $180^{\circ}$ ) domains with the account of surface energy.

\section{Surface energy}

Magnetic ordering is usually accompanied by appearance of spontaneous strains that result in change of the surface area of the sample. The related contribution to the free energy depends upon the spatial distribution of the strain field on the surface. Because of long-range (Coulomb) nature of surface forces it can be represented as follows:

\footnotetext{
*e-mail: malyshen@ukrpack.net
} 


$$
\begin{aligned}
F_{\text {surf }} & =\frac{1}{2} \int_{S} \mathrm{~d} S \int_{\boldsymbol{q}} \alpha(\boldsymbol{n}, \boldsymbol{q}) \mathrm{d} \boldsymbol{q}\left\{[\hat{u}(\boldsymbol{q}) \times n]^{2}+[\hat{\omega}(\boldsymbol{q}) \times n]^{2}+n[\hat{u}(\boldsymbol{q})-\hat{\omega}(q)]\right. \\
& \times[\hat{u}(q)+\hat{\omega}(q)] n-[n(\hat{u}(q)-\hat{\omega}(q)) n][n(\hat{u}(q)+\hat{\omega}(q)) n]\},
\end{aligned}
$$

where the sample suriace $S$ is defined (locally) by the unit normal $n, \hat{u}(\boldsymbol{q}), \hat{\omega}(\boldsymbol{q})$ are the $q$-th Fourier components of the strain tensor and antisymmetric tensor of rotations, respectively. Coefficient $\alpha(n, q)$ is the surface tension of the crystal face indicated by normal $\boldsymbol{n}$ that corresponds to the sinusoidal variation of strain $(\hat{u}(\boldsymbol{r})=\hat{u}(\boldsymbol{q}) \sin \boldsymbol{q} r)$ near the surface. It includes not only the contribution related with surface area increase, but also the energy related with bending of the surface. It was shown [2] that the forces appearing on the corrugated surface can give rise to the decrease in surface energy in comparison with the flat surface. Therefore, it can be naturally supposed that $\alpha(q)$ is an abruptly decreasing function of $q$.

\section{Thermodynamically equilibrium domain structure of AFM}

Let us consider a pure AFM with $N$-times degenerated equilibrium state characterized by the spontaneous strains $\hat{u}_{k}$, where $k=1,2, \ldots N$ (we do not differentiate between the states with opposite directions of AFM vector). Strain tensor $\hat{u}_{k}$ consists of isomorphous components that are equal for all the $k$ (e.g., bulk magnetostriction), and non-isomorphous (shear) components, $\hat{u}_{k}^{\text {shear }}$. From symmetry considerations it follows that $\sum_{k} \hat{u}_{k}^{\text {shear }}=0$.

The DS of the finite-size sample with characteristic size $L$ can be found from minimization of the full free energy of the crystal

$$
F=V f_{\text {homo }}+F_{\text {dw }}+F_{\text {surf }},
$$

where $V$ is the crystal volume, $f_{\text {homo }}$ is the density of volume free energy in homogeneous regions, $F_{\mathrm{dw}}$ accounts for the energy of the domain walls. The first term in (2) is independent of the DS. In the monodomain state the surface energy term can be rather high due to the contribution from the long-wavelength components with $q_{\min } \sim 2 \pi / L \rightarrow 0$. In the polydomain state this term could be minimized if the average shear strains (that coincide with the long-wavelength Fourier components, $\hat{u}^{\text {shear }}(0)=\sum_{k} \xi_{k} \hat{u}_{k}^{\text {shear }}=0, \xi_{k}$ is the volume fraction of the $k$-th domain) turn to zero. The sum of short-wavelength contribution from the surface energy and energy of the domain walls can also be minimized by a proper choice of the period of the DS $D=2 \pi / q_{\text {opt }}$. Really, $F_{\mathrm{dw}} \sim 1 / D$ and so, is increasing function of $q$, while $\alpha(q)$ is a decreasing function. The optimal value $q_{\circ \mathrm{opt}}$ can then be found from equation $\frac{\partial}{\partial q}\left(F_{\mathrm{dw}}+F_{\text {surf }}\right)=0$.

If the sample size is rather large $(L \gg D)$, so that $q_{\min } \ll q_{\text {opt }}$, then the polydomain state is energetically preferable in comparison with the monodomain one, because the energy growth resulting from the domain walls formation is compensated by the great advantage in surface energy related with zero average strains. This, particularly, means that formation of the domain structure is thermodynamically advantageous and for ideal crystal all the domains are equally represented, i.e. $\xi_{k}=1 / N$.

In opposite case, $L \leq D$, the loss in the energy of domain walls is not compensated by the advantage in the surface energy, therefore, the thermodynamically favorable state is the monodomain one. 


\section{The DS of pure AFM in the external magnetic field}

The behavior of the DS of pure AFM in the external magnetic field is analogous to that of ferromagnet [3]. Let us consider, for example, an easy-plane AFM with the magnetic field applied in the easy plane. The sample is supposed to be a single-crystal, polydomain, in the form of rotational ellipsoid with radius $R$ and principal axis directed perpendicular to the plane of magnetic ordering. Full free energy of the sample is given by the expression

$$
F=V\left\{\sum_{k} \xi_{k}\left[f_{\mathrm{an}}+\frac{M_{0}}{2 H_{\mathrm{E}}}\left(\boldsymbol{H} l_{k}\right)^{2}+\hat{u}_{k} \hat{\lambda} l_{k} \otimes l_{k}+\frac{1}{2} \hat{u}_{k} \hat{c} \hat{u}_{k}\right]+\frac{\langle\hat{u}\rangle \alpha\langle\hat{u}\rangle}{2 R}\right\},
$$

where $f_{\text {an }}$ is the magnetic anisotropy energy, $M_{0}$ is saturation magnetization, $2 H_{\mathrm{E}}$ and $H$ are the spin-flip and external magnetic fields, respectively, $l_{k}$ is the vector of AFM in the $k$-th domain, $\hat{\lambda}, \hat{c}$ are the tensors of magnetoelastic and elastic coefficients, respectively, $\langle\hat{u}\rangle=\sum_{k} \xi_{k} \hat{u}_{k}$ is the strain tensor averaged over the sample volume and corresponding to zero Fourier component $\hat{u}^{\text {shear }}(0)$. In the expression (3) we have neglected demagnetization energy which is immaterial for further consideration.

Last term in (3) arises from long-wavelength component of the surface energy. The 4th rank tensor $\hat{\alpha}$, symmetrical over the pairs of indices, is defined by the shape of the sample

$$
\alpha_{i p j l}=\frac{1}{S} \int_{S} \mathrm{~d} S \alpha(n, 0)\left[e_{i j k} e_{p l m} n_{k} n_{m}+n_{i} n_{j} \delta_{p l}+n_{p} n_{l} \delta_{i j}-n_{i} n_{p} n_{j} n_{l}\right]
$$

( $e_{i j k}$ is completely antisymmetric unit tensor). It can be treated as a form tensor, analogous to the tensor of demagnetization for the ferromagnet.

In principle, the external magnetic field produces the processes of rotation and domain wall shifting. In the case of mobile (unpinned) domain walls, the surface tension that depends upon the relative fraction of the domains causes a kind of "demagnetization" field through the magnetoelastic interactions. An effective magnetic field, $H_{\text {eff }}$ in the sample can then be calculated from the equations

$$
\boldsymbol{H}_{\mathrm{eff}} \otimes \boldsymbol{H}_{\mathrm{eff}}=\boldsymbol{H} \otimes \boldsymbol{H}+\frac{2 H_{\mathrm{E}}}{M_{0}} \sum_{k} \xi_{k} l_{k} \otimes l_{k}\left[\hat{\lambda}(\hat{c} R+\hat{\alpha})^{-1} \hat{\alpha} \hat{c}^{-1} \hat{\lambda}\right] .
$$

If the value of external field is rather small (see below), the external magnetic field is fully compensated due to the redistribution of the domains. The effective magnetic field inside the sample is zero, therefore, the possible values of AFM vectors and spontaneous strains coincide with their equilibrium zero-field values. The relative fractions of the domains can be defined from the condition $\boldsymbol{H}_{\text {eff }}=0$ as follows:

$$
\xi_{k}=\frac{1}{n}\left[1+\frac{H^{2} \boldsymbol{l}_{k}^{2}-2\left(\boldsymbol{H} l_{k}\right)^{2}}{H_{\mathrm{md}}^{2}}\right], \quad k=1,2, \ldots, N
$$

where $H_{\mathrm{md}} \equiv \lambda M_{0}^{2} \sqrt{\alpha H_{\mathrm{E}}} / \sqrt{M_{0} c(c R+\alpha)}$ is the characteristic field of monodomenization. (Here $\lambda, \alpha$, and $c$ are the certain combinations of magnetoelastic, elastic, and surface tension constants that should be calculated with the account of concrete symmetry of the crystal.) It can be easily seen that in the case of zero effective 
field the specific free energy (chemical potential) of different domains (phases) is equal, therefore, these domains can coexist in thermodynamic equilibrium.

According to (5), the volume fraction of the domains depends upon the value of the external magnetic field $H$. At zero field, $H=0$, all types of the domains are equally represented, so that the symmetry of the sample does not change after the transition into the polydomain AFM state. In nonzero field the fraction of the most energetically "unfavorable" domain (with the AFM vectors, say $l_{1}$, lying closely to the direction of the magnetic field), diminishes. At $H=H_{\mathrm{md}} / \sqrt{\cos 2 \psi_{1}}$ ( $\psi_{1}$ is an angle between $\boldsymbol{H}$ and $\boldsymbol{l}_{1}$ ) the "unfavorable" domain disappears. At $H>H_{\mathrm{md}} / \sqrt{\cos 2 \psi_{1}}$, the effective magnetic field in the sample is nonzero and rotational processes take place along with the shift of the domain walls. The description of the DS in this case needs the specification of the crystal structure and is out of scope of this paper.

From (6) and (3) the experimentally measurable relative elongation of the AFM sample in the direction of the external magnetic field, $(\Delta \ell / \ell)_{\|}$, can be calculated as follows:

$$
\left(\frac{\Delta \ell}{\ell}\right)_{\|}=-\frac{R \lambda M_{0}^{2}}{c R+\alpha}\langle\cos 2 \psi\rangle=-\frac{R \lambda M_{0}^{2} H^{2}}{2 H_{\mathrm{md}}^{2}(c R+\alpha)} .
$$

For small field values the magnetostriction of the sample linearly depends upon the $H^{2}$ value independently of the orientation of crystal axes with respect to the field direction. Such kind of dependence was observed in [1] for different easy-plane pure antiferromagnets of $\mathrm{CoCl}_{2}$-type.

\section{Conclusions}

1. Formation of the domain structure in pure AFM with non- $180^{\circ}$ is thermodynamically favorable due to the influence of the surface energy related with the spontaneous magnetoelastic strains. The effect of surface tension can be described by the form-tensor (4), analogous to the tensor of demagnetization for ferromagnets.

2. The formation of the DS is unfavorable in small samples, the critical size is defined by the values of surface tension and energy of domain walls.

3. If the most of domain walls are mobile, the DS is reversible in the external magnetic field. For small field value, redistribution of the domains induced by the surface tension, results in compensation of the external field. As a result, magnetostriction of the sample linearly depends upon the $H^{2}$ value up to the field of monodomenization.

\section{References}

[1] V.M. Kalita, A.F. Losenko, S.M. Ryabchenko, P.A. Trotsenko, Ukr. Fiz. Zh. 43, 1469 (1998).

[2] V.I. Marchenko, Zh. Eksp. Teor. Fiz. 80, 257 (1980).

[3] L. Néel, J. Phys. Rad. (France) 5, 241 (1944). 